\title{
CHANCE AND NON-CHANCE CLUSTERING IN THE UNIVERSE AND PROBLEM OF HIGH REDSHIFT GALAXIES IN COMPACT GROUPS.
}

\author{
JOANNA ANOSOVA ${ }^{1}$, LUDMILA KISELEVA ${ }^{2}$ \\ ${ }^{1}$ National Astronomical Observatory, Tokyo 181, Japan. \\ ${ }^{2}$ Institute of Astronomy, University of Cambridge, \\ Madingley Road, Cambridge CB3 OHA, England.
}

\section{Introduction}

In previous work (Anosova, Kiseleva 1993) we have studied the statistical properties of Hickson's (1982) compact galaxy groups. We have shown that most of these groups are confident and probable physical systems of galaxies. Only about $13 \%$ of galaxies can be non-members of their groups due to the effects of projection.

In this work we apply a new classification technique to the galaxies which have concordant and discordant redshifts in compact groups. We define the probability that these galaxies are not chance objects inside their groups. Usually authors consider independently the angular separations $\rho$ and differences of radial velocities $d V$ of galaxies under study. Then they use various critical values of $d V_{c r}$. In our method $\rho$ and $d V$ are used simultaneously. We define the probability $P$ and expectation $E X$ of a number of chance groups with similar relative observed data for the components on the sky sphere (a random uniform distribution is assumed for field galaxies). We have classified the galaxies from compact groups into four classes:

I. confident non-chance groups,

II. probable non-chance groups,

III. probable chance groups,

IV. confident chance groups.

If the redshifts indicate the distances of the galaxies from the Sun, close non-chance groups of galaxies are systems with physically connected components; the discordant members of wide non-chance groups are con-

M. Kafatos and Y. Kondo (eds.), Examining the Big Bang and Diffuse Background Radiations, 511-515.

(c) 1996 IAU. Printed in the Netherlands. 
fident single galaxies belonging to voids (between members of wide nonchance groups there are almost empty regions). Chance groups are fortuitous groupings of field galaxies and may be called 'optical' ones.

\section{Classification and basic characteristics of chance and non- chance pairs}

In this work, we develop a new method for the determination of structures of fields of astronomical objects and apply this method to the observed distribution of galaxies in the Universe. Using the theory of probability, we carry out a quantative statistical analysis and find parameters which characterize differences in the distributions of galaxies in the sky and objects in a model uniform random field. In the case of galaxies, we consider the cut phase space taking into account simultaneously the angular separations $\rho$ between galaxies, and their radial velocities $V$. We assume that the radial velocities of these objects indicate their Hubble distances, and we do not consider the peculiar velocities of galaxies.

The following classification of the objects is used:

1. members of clusters with different multiplicities;

2. uniform random distributed single objects in the galaxy field;

3. objects separated by voids.

We suggest a definition of the probability $P_{n c h}$ for objects to belong to these classes, and the critical values of $P_{n c h}$ for this classification. The relative uncertainties in the observations can be take into account.

Our statistical analysis involves the number $N=4.5 \times 10^{7}$ of objects in the celestial sphere with radius corresponding to the maximum velocity $V_{\max }=45000 \mathrm{~km} / \mathrm{s}$ of galaxies from CfA-Catalogue. We take the mean number density of galaxies in the field $\nu=0.05 \mathrm{gal} / \mathrm{Mpc}^{3}$ and the Hubble constant $H=75 \mathrm{~km} / \mathrm{s} / \mathrm{Mpc}$. For every object under consideration, we calculate the probability $P_{n c h}$ and identify the confident and probable members of galaxy groups and clusters.

The formula for probability $P_{n}$ of a chance realization of a group with multiplicity $n$, and for expectation $E X_{n}$ of the number of such groups inside the whole sky sphere, were given by Anosova (1987) and Anosova \& Kiseleva (1993), and include two important terms: (a) $B^{n-1}$ and (b) $(1-B)^{N-n}$. $B=V(\varsigma) / V\left(\sum\right), V\left(\sum\right)$ and $V(\varsigma)$ are the volumes of $\sum$ and $\varsigma, \varsigma$ being the phase space occupied by a group, while $\sum$ is the phase space of the whole sample of $N$ objects.

The term $B^{n-1}$ means that the given $n$ objects occur in the region $\varsigma$ by chance, and the term $(1-B)^{N-n}$ indicates that there are no other objects $N-n$ in the same region. Therefore, the proposed method can be 
used for the identification of clusters of objects as well as of voids in the Metagalactic field.

One can expect that with an increase of the phase volume occupied by the group, the values of $P(n)$ and $E X(n)$ also increase initially, reach their maxima, and then decrease till $P(n) \approx 0, E X(n)<1$.

For every phase space volume $\Sigma(R)$ with a radius $R$ and the assumed average number density $\nu$ of the objects, we can find the maximal number

$$
E X_{\max }(n)=E X_{c h}
$$

of chance groups with a given multiplicity $n$. In order to obtain the typical basic characteristics of such chance groups the following modeling procedure was performed.

For the definition of $E X_{\max }(n)$ we consider a model of the galaxy field which is the phase space sphere $\Sigma(R)$ of radius $R$ corresponding to the largest radial velocity $V$ of galaxies in this field. We examine the galaxy fields with various $V$ corresponding to the observed data for the CFA galaxies: $1000 \leq V \leq 45000 \mathrm{~km} / \mathrm{s}$. Using the expression $N=4 / 3 \pi \nu R^{3}$, for every $V$ we find the total number $N$ of galaxies inside this field. We construct model pairs $(n=2)$ of galaxies with various values of angular separations $\rho$ and differences of radial velocities $d V$ : we scan $d V$ and $\rho$ in the following intervals:

(a) we choose $d V$ such that $d V / V$ lies in the interval $(0.0,1.0)$

(b) in order to obtain typical values of the parameters for galaxy pairs of all classes I-IV we examined a large range of angular separations between galaxies from $0^{\circ}$ to $50^{\circ}$.

Table I shows the results for pairs of galaxies of the model fields: for a given $V$, this Table contains the number $N$ of galaxies in the phase space $\Sigma(R)$, the values $E X_{c h}=E X_{\max }(2)(n=2)$, as well as the average values $d V_{c h}$ and $\rho_{c h}$ and their r.m.s. deviations for confident chance pairs of galaxies. The last column in Table I gives the maximal possible angular separation $\rho$ between two physicaly connected galaxies for given $V$. A detailed description of a new method for classification of galaxies from any field into eight classes (I-VIII) between confident clusters and confident objects separated by voids was given by Anosova et al. (1994).

\section{Results for compact groups}

As an extention of our previous work (Anosova \& Kiseleva 1993), in the present work we apply our new algorithm for the identification of chance and non-chance clusters and voids to Hickson's compact galaxy groups. These groups contain galaxies with concordant and discordant redshifts (Table 2). 
TABLE 1. Characteristics of typical chance pairs

\begin{tabular}{cccccccc}
\hline$V$ & $N$ & $E X_{c h}$ & $d V_{c h}$ & $\delta_{d} V_{c h}$ & $\rho_{c h}$ & $\delta \rho_{c h}$ & $\rho_{c l}$ \\
\hline 1000 & $5.0 \times 10^{2}$ & 91 & 98 & 85 & 898 & 278 & 374 \\
2000 & $4.0 \times 10^{3}$ & 730 & 106 & 88 & 319 & 101 & 133 \\
3000 & $1.3 \times 10^{4}$ & $2.5 \times 10^{3}$ & 162 & 138 & 174 & 55 & 72 \\
5000 & $6.2 \times 10^{4}$ & $1.1 \times 10^{4}$ & 226 & 190 & 80 & 25 & 34 \\
$6000 *$ & $1.1 \times 10^{5}$ & $2.0 \times 10^{4}$ & 262 & 222 & 61 & 19 & 25 \\
$8000 *$ & $2.5 \times 10^{5}$ & $4.7 \times 10^{4}$ & 326 & 276 & 40 & 12 & 16 \\
10000 & $5.0 \times 10^{5}$ & $9.1 \times 10^{4}$ & 387 & 327 & 29 & 9 & 12 \\
$20000 *$ & $4.0 \times 10^{6}$ & $7.3 \times 10^{5}$ & 666 & 562 & 10 & 2 & 4.1 \\
$35000 *$ & $2.1 \times 10^{7}$ & $3.9 \times 10^{6}$ & 1050 & 886 & 4.5 & 1.5 & 1.9 \\
40000 & $3.2 \times 10^{7}$ & $5.8 \times 10^{6}$ & 1172 & 988 & 3.6 & 1.2 & 1.5 \\
45000 & $4.5 \times 10^{7}$ & $8.3 \times 10^{6}$ & 1289 & 1087 & 3.0 & 1.2 & 1.3 \\
\hline
\end{tabular}

TABLE 2. Classification of galaxies from compact groups

I. Concordant redshifts $\left(d V<10^{3} \mathrm{~km} / \mathrm{s}\right)$ :

1. confident non-chance members of clusters, $N_{\text {gal }}=40$;

$10^{-5}<P_{c h}<10^{-3}, d V<500 \mathrm{~km} / \mathrm{s} ;$

2. probable non-chance members of clusters, $N_{\text {gal }}=244$;

$10^{-3}<P_{c h}<10^{-2}, 500<d V<10^{3} \mathrm{~km} / \mathrm{s}$;

II. Discordant redshifts $\left(d V>10^{3} \mathrm{~km} / \mathrm{s}\right)$ :

1. 'bright discordants', $N_{\text {gal }}=11$;

$10^{-3}<P_{c h}<10^{-2}, 10^{3}<d V<10^{4} \mathrm{~km} / \mathrm{s}$;

2. 'double chance groups', $N_{g r}=6, N_{\text {gal }}=34$;

$10^{-3}<P_{c h}<10^{-1}, 6 \times 10^{3}<d V<11 \times 10^{3} \mathrm{~km} / \mathrm{s}$

3. probable chance 'groups', $N_{\text {gal }}=24$;

$10^{-1}<P_{c h}<0.20, \times 10^{3}<d V<18 \times 10^{3} \mathrm{~km} / \mathrm{s}$

4. confident chance 'groups', $N_{\text {gal }}=10$;

$0.26<P_{c h}<0.98,8 \times 10^{3}<d V<19 \times 10^{3} \mathrm{~km} / \mathrm{s}$;

We find that $97 \%$ of galaxies are not by chance in the phase space of their groups with probability $P_{n c h}>0.80$. Another $3 \%$ of galaxies can be objects, from a field with random uniform distribution of galaxies, which are projected on to the groups. Most of the discordant galaxies had been identified as confident discordants in our previous work, but other types of discordant galaxies appear to have non-random connection with their groups; possibly these objects are separated by voids.

We can now redetermine multiplicities of compact groups, and we find that 7 of them are binary and 13 are triple galaxies (Hickson defined for 
his groups the multiplicity $n \geq 4$ ); the number of quartets is reduced from 59 to 55 , the number of quintets from 26 to 22 , of sextets from 7 to 4 , and the number of groups with $N=7$ from 6 to 4 .

Thus we can conclude that the majority of the compact groups examined are confident non-chance systems of galaxies. We can suggest some possible explanations for confident non-chance members of compact groups with discordant redshifts:

- objects separated by voids;

- products of strong dynamical interaction between galaxies within groups;

- non-Hubble distances or other physical reasons.

Therefore, components of compact groups of galaxies with discordant redshifts require a special study. An application of redshift-independent methods of distance determination may be especially important in these cases.

Our results agree with the conclusions of Hickson and Sulentic (Sulentic 1987 , 1988; Hickson 1990) that only about $3 \%$ of the compact groups are confident chance ones.

\section{References}

Anosova J.P., 1987, Astrofizika, 27, 535.

Anosova, J.P., Kiseleva, L.G., 1993, Astrophys. Space Sc., 209, 181.

Anosova, J.P., Iyer, S., Varma, R.K., 1994, Ap.J., submitted.

Hickson, P.,1982, Astrophys. J., 255, 382.

Hickson, P., 1990, Proc. IAU Coll. No. 124, 77.

Sulentic, J.W., 1987, Astrophys. J., 322, 605.

Sulentic, J.W., 1988, in New ideas in Astronomy, ed. F. Bertola, J. Sulentic, B. Madore, 123. 